\title{
Delivery of the Sox9 gene promotes chondrogenic differentiation of human umbilical cord blood-derived mesenchymal stem cells in an in vitro model
}

\author{
Z.H. Wang ${ }^{1}$, X.L. Li ${ }^{2}$, X.J. He ${ }^{3}$, B.J. Wu ${ }^{1}$, M. Xu' ${ }^{1}$, H.M. Chang ${ }^{4}$, X.H. Zhang ${ }^{1}$, Z. Xing ${ }^{5}$, X.H. Jing ${ }^{1}$, \\ D.M. Kong ${ }^{1}$, X.H. Kou ${ }^{1}$ and Y.Y. Yang ${ }^{1}$
}

${ }^{1}$ Department of Otolaryngology - Head and Neck Surgery, The Second Hospital, Xi'an Jiaotong University, Xi'an, China ${ }^{2}$ Department of Dermatology, The Second Hospital, Xi'an Jiaotong University, Xi'an, China

${ }^{3}$ Department of Orthopedics, The Second Hospital, Xi'an Jiaotong University, Xi'an, China ${ }^{4}$ Department of Otolaryngology - Head and Neck Surgery, Affiliated Hospital of Xi'an Medical University, Xi'an, China ${ }^{5}$ Department of Clinical Dentistry, Faculty of Dentistry, Center for Clinical Dental Research, University of Bergen, Bergen, Norway

\begin{abstract}
SRY-related high-mobility-group box 9 (Sox9) gene is a cartilage-specific transcription factor that plays essential roles in chondrocyte differentiation and cartilage formation. The aim of this study was to investigate the feasibility of genetic delivery of Sox9 to enhance chondrogenic differentiation of human umbilical cord blood-derived mesenchymal stem cells (hUC-MSCs). After they were isolated from human umbilical cord blood within $24 \mathrm{~h}$ after delivery of neonates, hUC-MSCs were untreated or transfected with a human Sox9-expressing plasmid or an empty vector. The cells were assessed for morphology and chondrogenic differentiation. The isolated cells with a fibroblast-like morphology in monolayer culture were positive for the MSC markers CD44, CD105, CD73, and CD90, but negative for the differentiation markers CD34, CD45, CD19, CD14, or major histocompatibility complex class II. Sox9 overexpression induced accumulation of sulfated proteoglycans, without altering the cellular morphology. Immunocytochemistry demonstrated that genetic delivery of Sox9 markedly enhanced the expression of aggrecan and type II collagen in hUC-MSCs compared with empty vector-transfected counterparts. Reverse transcriptionpolymerase chain reaction analysis further confirmed the elevation of aggrecan and type II collagen at the mRNA level in Sox9transfected cells. Taken together, short-term Sox9 overexpression facilitates chondrogenesis of hUC-MSCs and may thus have potential implications in cartilage tissue engineering.
\end{abstract}

Key words: Genetic modification; Tissue engineering; Stem cells; Sox9; Chondrogenesis

\section{Introduction}

Cartilage regeneration is often needed in orthopedic or plastic surgery for the repair of cartilaginous defects. However, due to the limited regenerative capacity of cartilage tissue, the treatment of various cartilaginous lesions remains a challenge to clinicians. The focal treatment strategies for osteochondral defects are currently associated with a variety of risks and limitations including inadequate availability of donor tissues, donor site morbidity, and poor attachment of the graft to the surrounding chondral surface (1). Tissue engineering has emerged as a promising new method for cartilage repair in which a combination of cells, scaffolding, and bioactive agents are used to fabricate functionally engineered cartilage tissue (2).

The cell source is an important factor for successful tissue engineering, and chondrocytes that can be expanded in vitro have been commonly used in cartilage tissue engineering (3). However, the relatively low availability and proliferation potential of chondrocytes hamper their application in tissue engineering. In vitro

Correspondence: B.J. Wu, Department of Otolaryngology - Head and Neck Surgery, The Second Hospital, Xi'an Jiaotong University, Xi'an 710004, China. Fax: + 86-029-8767-8421. E-mail: ehui4298@163.com and Z. Xing, Department of Clinical Dentistry, Faculty of Dentistry, Center for Clinical Dental Research, University of Bergen, Bergen, Norway. E-mail: Zhe.xing@iko.uib.no 
expansion is accompanied by chondrocyte dedifferentiation, resulting in substantial molecular and phenotypic changes (4). Dedifferentiated chondrocytes show decreased proteoglycan synthesis and type II collagen expression and increased type I collagen expression, thus failing to produce a mechanically normal cartilage extracellular matrix (ECM).

In addition to chondrocytes, stem cells have also been explored for the repair of damaged cartilage (5). Mesenchymal stem cells (MSCs) are a population of multipotent cells that can differentiate into different cellular lineages including not only osteoblasts, chondrocytes, and adipocytes but also muscle cells, cardiomyocytes, and neural precursors (6-8). MSCs have been identified in a broad range of tissues including bone marrow, adipose tissue, synovial tissue, and umbilical cord blood (9). Umbilical cord blood is an important source of human MCSs, and the isolation of MSCs from umbilical cord has potential advantages over isolation from bone marrow, including simplicity, cost effectiveness, and noninvasiveness. Moreover, human umbilical cord blood-derived MSCs (hUC-MSCs) are poorly immunogenic and show immunosuppressive effects $(10,11)$, thereby facilitating graft tolerance.

Because the incidence of spontaneous chondrogenic differentiation of MSCs is very low, many pharmacological and genetic approaches have been developed to induce such differentiation (12). SRY-related high-mobility-group box 9 (Sox9) gene is a cartilage-specific transcription factor and plays essential roles in chondrocyte differentiation and cartilage formation (13). Sox9 is responsible for the expression of several cartilage-specific ECM components including aggrecan and collagens II, IX, and XI (14), and compelling evidence indicates that Sox9 is involved in chondrogenesis of MSCs $(15,16)$. Kawakami et al. (15) reported that overexpression of Sox9 and its coactivator (i.e., peroxisome proliferator-activated receptor gamma coactivator 1-alpha) induces expression of chondrogenic genes, followed by chondrogenesis in MSCs. The delivery of Sox9 was found to enhance chondrogenic differentiation but to decrease osteogenic and/or adipogenic differentiation in human bone marrow-derived MSCs (16).

Despite many studies on the committed differentiation of bone marrow-derived MSCs, relatively less attention has been paid to promotion of chondrogenesis in hUCMSCs. Given the master role of Sox9 in chondrogenesis, in the present study we investigated the feasibility of genetic delivery of Sox9 to enhance chondrogenic differentiation of hUC-MSCs.

\section{Material and Methods}

\section{Isolation of hUC-MSCs}

Human umbilical cords were obtained and processed within $24 \mathrm{~h}$ after delivery of neonates. All procedures were approved by the Ethics Committee of Xi'an Jiaotong
University (China). Umbilical cord blood samples were diluted 1:1 in phosphate-buffered saline (PBS) and mixed with $3 \%$ gelatin to deplete red blood cells. The plasma fraction was collected and centrifuged at $2500 \mathrm{~g}$ for $5 \mathrm{~min}$, and the cellular pellet was resuspended in alpha-minimum essential medium ( $\alpha$-MEM). The cell suspension was transferred to centrifuge tubes containing twice the volume of Ficoll-Paque solution (Sigma, USA) at a density of $1.077 \mathrm{~g} / \mathrm{mL}$, and subjected to centrifugation at $2500 \mathrm{~g}$ for 20 min to isolate the fraction of mononuclear cells that contained MSCs (17). The isolated cells were washed twice with D-Hank's buffer and cultured at a density of $1 \times 10^{6}$ cells $/ \mathrm{cm}^{2}$ in $\alpha$-MEM containing $20 \%$ fetal bovine serum (FBS) and 1\% antibiotic/antimycotic (Sigma). The relatively high plating density facilitates rapid growth and expansion and assists cell survival. The culture medium was changed every 2 days, and cells were subcultured when they reached about $50 \%$ confluence.

\section{Cell proliferation assay}

To evaluate the effect of cryopreservation on the proliferation potential of hUC-MSCs, cells at passage 3 were pelleted, resuspended in $\alpha$-MEM containing $20 \%$ FBS and $10 \%$ dimethyl sulfoxide, and cryopreserved in liquid nitrogen for 3 months. After thawing, the cryopreserved cells were seeded at a density of $1 \times 10^{4}$ cells/well onto 24-well plates and cultured in $\alpha$-MEM supplemented with $20 \%$ FBS. Noncryopreserved cells were also cultured under the same conditions as described above. The adherent cells were counted daily for 8 days, and growth curves were plotted as total cell number vs time.

\section{Immunophenotyping of hUC-MSCs by flow cytometry}

Freshly isolated hUC-MSCs were harvested by treatment with $0.1 \%$ trypsin-EDTA, and detached cells were washed with PBS and incubated at $4{ }^{\circ} \mathrm{C}$ for $30 \mathrm{~min}$ with the following mouse anti-human antibodies: anti-CD34, -CD44, -CD45, -CD105, -CD73, -CD90, -CD19, -CD14, and major histocompatibility complex class II (MHC II). These antibodies were conjugated with either fluorescein isothiocyanate (FITC) or phycoerythrin (PE) (both from Becton Dickinson, USA). FITC- or PE-conjugated IgG1 was used as isotype control. After they were washed, labeled cells were assayed by flow cytometry (Becton Dickinson).

\section{Transfection of Sox9-expressing plasmids}

The hUC-MSCs were seeded at $5 \times 10^{5}$ cells per well on 6 -well plates. At $80-90 \%$ confluence, the cells were transfected with an empty vector or Sox9-expressing plasmid with a green fluorescent protein (GFP) tag at the $\mathrm{N}$-terminal end using Lipofectamine 2000 reagent, according to the manufacturer's instructions (Invitrogen, USA). After $6 \mathrm{~h}$ of transfection, the medium containing transfection reagents was removed and fresh culture medium containing $10 \%$ FBS was added to the cells. 
Transfection efficiency was determined by estimating the percentage of GFP-positive cells under fluorescence microscopy $48 \mathrm{~h}$ posttransfection.

\section{Chondrogenic differentiation of hUC-MSCs}

hUC-MSCs at passage 3 were used to induce chondrogenic differentiation. The cells were divided into four groups: untreated control group, differentiationinduced (DI) group, Sox9 group, and empty vector group. In the DI group, cells were cultured in chondrogenic medium (18) containing high-glucose Dulbecco's modified Eagle's medium, 5\% FBS, $100 \mathrm{nM}$ dexamethasone, $50 \mu \mathrm{g} / \mathrm{mL}$ ascorbate-2-phosphate, $10 \mathrm{ng} / \mathrm{mL}$ recombinant transforming growth factor- $\beta, 10 \mathrm{ng} / \mathrm{mL}$ recombinant insulin-like growth factor-I, and ITS ${ }^{+}$Premix (Sigma). In the Sox9 and empty vector groups, hUC-MSCs were transfected with Sox9-expressing plasmid and empty vector, respectively, in the presence of G418 (600 $\mu \mathrm{g} / \mathrm{mL}$ ). After incubation for 10 days, the cells were transferred to G418-free medium and cultured for another 11 days. Chondrogenic differentiation was assessed by toluidine blue (Sigma) staining.

\section{Reverse transcription-polymerase chain reaction (RT- PCR)}

Total RNA was extracted from treated and untreated cultures using TRIzol reagent according to the manufacturer's protocol (Invitrogen). First-strand cDNA was synthesized using the PrimeScript RT-PCR reagent kit (Takara, China), according to the manufacturer's instructions. The specific primers used for RT-PCR are shown in Table 1. $\beta$-actin was amplified as an internal control for normalization. PCR products were separated on $1.5 \%$ agarose gels and visualized by ethidium bromide staining (19), and the images were analyzed by the GEL DOC 2000 system (Bio-Rad, USA), where relative expression level (\%) equaled gene band density divided by $\beta$-actin band density.

\section{Immunocytochemistry}

Cells were fixed in $4 \%$ paraformaldehyde for $30 \mathrm{~min}$ and treated with methanol and $0.1 \%$ Triton $\mathrm{X}-100$ to achieve cell membrane and nuclear membrane permeability (20). Nonspecific binding was blocked by incubation with normal goat serum for $30 \mathrm{~min}$. The hUC-MSCs were incubated with 1:200 mouse anti-human collagen II or goat anti-human aggrecan antibody overnight at $4^{\circ} \mathrm{C}$, and biotinylated secondary antibody was applied for $30 \mathrm{~min}$ at room temperature. After they were thoroughly washed with PBS containing $1 \%$ bovine serum albumin, the cells were incubated with horseradish peroxidase (HRP)-labeled streptavidin (ABC kit, Vector Laboratories, USA), followed by reaction with diaminobenzidine (Sigma). A negative control was included without addition of primary antibody. Cells were photographed with an Olympus IX 70 microscope, and gray density was analyzed using an image analysis system (Leica, Germany).

\section{Western blotting analysis}

Cells were lysed in lysis buffer [50 mM Tris- $\mathrm{HCl}, \mathrm{pH} 7.4$, $150 \mathrm{mM} \mathrm{NaCl}, 1 \%$ nonylphenoxypolyethoxyethanol (NP)40 , and $0.1 \%$ sodium dodecyl sulfate (SDS) supplemented with protease and phosphatase inhibitors]. The protein extracts were separated on $12 \%$ polyacrylamide gels containing $0.1 \%$ SDS and then transferred to a nitrocellulose membrane. After it was blocked for $4 \mathrm{~h}$ in buffer containing $5 \%$ fat-free dried milk and $0.5 \%$ Tween-20, the membrane was incubated with anti-collagen II monoclonal antibody or anti- $\beta$-actin polyclonal antibody overnight at $4^{\circ} \mathrm{C}$. The membrane was washed three times and incubated for $1 \mathrm{~h}$ with HRP-conjugated goat anti-rabbit IgG (dilution 1:7000)

Table 1. Primers used for RT-PCR.

\begin{tabular}{|c|c|c|}
\hline Primer & Sequence & Product size (bp) \\
\hline Collagen II & & 138 \\
\hline Forward & 5'-GGAGCAGCAAGAGCAAGGAGAAG-3' & \\
\hline Reverse & 5'-TGGACAGCAGGCGTAGGAAGG-3' & \\
\hline Collagen I & & 235 \\
\hline Forward & 5'-CTTGGTCTCGTCACAGATCA-3' & \\
\hline Reverse & 5'-CTTTTAACGGAGGATGTGCTATTTGGC-3' & \\
\hline Aggrecan & & 309 \\
\hline Forward & 5'-GCAGTCTTCCAACCCAA-3' & \\
\hline Reverse & 5'-ACATCTCCAGCCTCCTTA-3' & \\
\hline Sox9 & & 631 \\
\hline Forward & 5'-GAACGCACATCAAGACGGAG-3' & \\
\hline Reverse & 5'-TCTCGTTGATTTCGCTGCTC-3' & \\
\hline$\beta$-Actin & & 179 \\
\hline Forward & 5'-ATCGTGCGTGACATTAAGGAGAAG-3' & \\
\hline Reverse & 5'-AGGAAGGAAGGCTGGAAGAGTG-3' & \\
\hline
\end{tabular}


or anti-mouse lgG (dilution 1:8000) at room temperature. The signals were visualized with the enhanced chemiluminescence method and developed on X-ray film. The band density was measured by the GEL DOC 2000 system equipped with the Quantity One software (Bio-Rad) and normalized against the density of $\beta$-actin.

\section{Statistical analysis}

Data are reported as means $\pm S D$ from three independent experiments and were evaluated by one-way analysis of variance followed by the Tukey multiple comparison test. A difference was defined as significant at $P<0.05$. All analyses were carried out using the SPSS 10.0 statistical software (SPSS, USA).

\section{Results}

\section{Morphology and phenotype characteristics of umbilical cord blood-derived cells}

When grown in monolayer culture, the cells isolated from umbilical cord blood initially exhibited a spindleshaped or polygonal morphology (Figure 1A). After five passages, the cell culture had a predominantly fibroblastlike morphology and often formed a squamous eddy-like structure (Figure 1B). Phenotypic characterization revealed that the cells were positive for several MSC markers including CD44, CD105, CD73, and CD90, but negative for the differentiation markers CD34, CD45,
CD19, CD14, or MHC II (Figure 1C). Compared with noncryopreserved cells, the cryopreserved counterparts had a slightly longer latency period and lower proliferation index (Supplementary Figure S1). However, the differences were not statistically significant $(P>0.05)$, and they had a comparable doubling time.

\section{Effects of Sox9 overexpression on morphology and chondrogenesis of hUC-MSCs}

Next, we examined the effects of enforced expression of Sox9 on the morphology and chondrogenesis of hUCMSCs. The transfection efficiency (as determined by the percentage of GFP-positive cells $48 \mathrm{~h}$ after transfection) was estimated to be about $80 \%$ (Supplementary Figure S2). Cells transfected with either empty vector or Sox9expressing plasmid had a fibroblast-like morphology similar to untreated control cells, without evident detachment (Figure 2A). In contrast, cells cultured in the chondrogenic medium were polygonal or irregular in shape and prone to detach from the plate (Figure 2A).

After 2-3 weeks of monolayer culture, Sox9-transfected hUC-MSCs and those treated with chondrogenic medium showed a similar accumulation of sulfated proteoglycans by toluidine blue staining (Figure 2B). However, toluidine blue staining was weak in untreated and empty vectortransfected control cells cultured in basic maintenance medium, suggesting the absence of chondrogenic differentiation (Figure 2B).
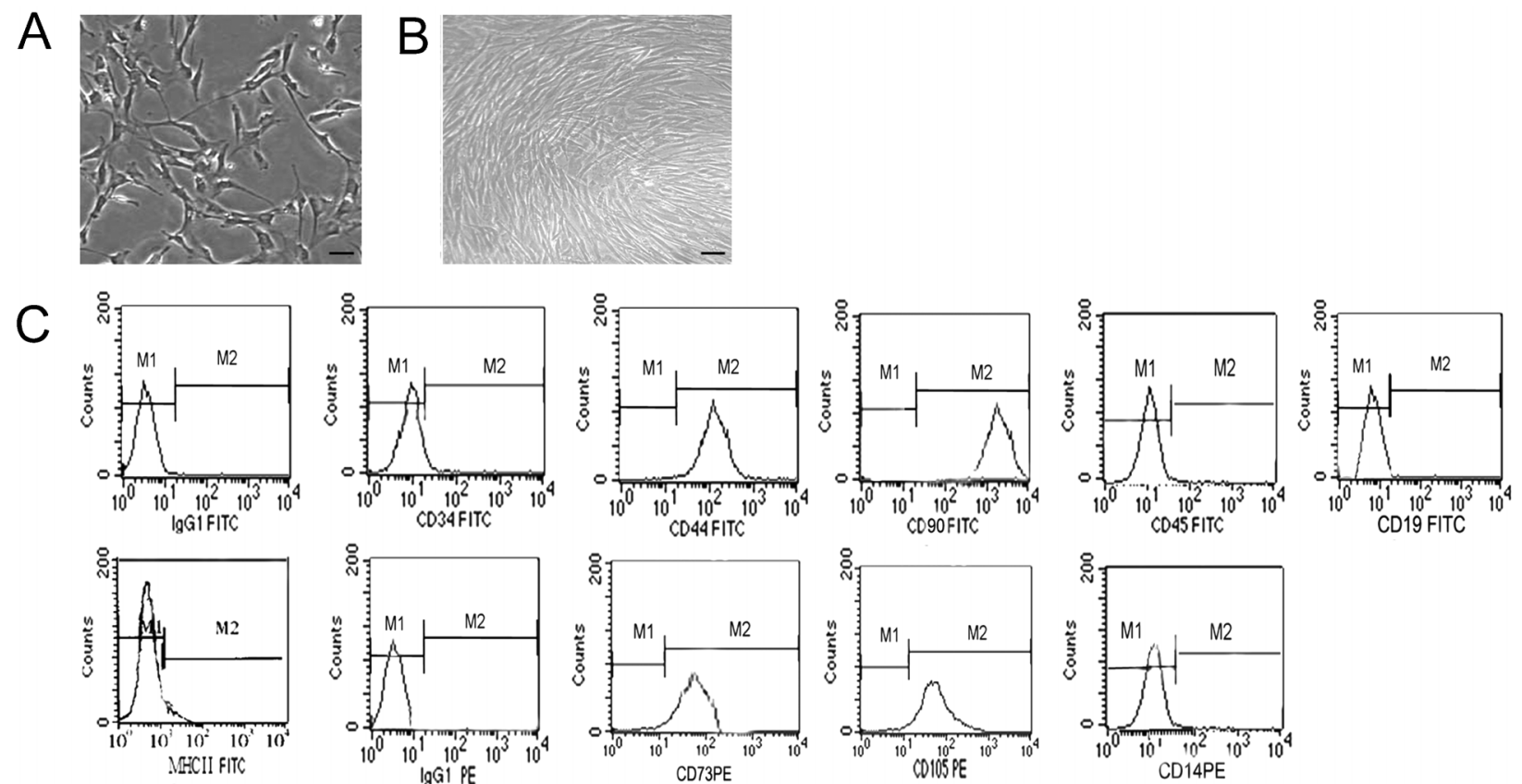

Figure 1. Characterization of hUC-MSCs. A, hUC-MSCs at passage 1 displayed a spindle-shaped or polygonal morphology in monolayer culture. $B$, hUC-MSCs at passage 5 became a predominant fibroblast-like morphology and formed a squamous eddy-like structure. Bar $=50 \mu \mathrm{m}$. C, Flow cytometric analysis of surface antigen markers. hUC-MSCs expressed CD44, CD105, CD73, and CD90, but not CD34, CD45, CD19, CD14, or MHC II. PE- and FITC-conjugated mouse monoclonal IgG1 were used as isotype controls. 


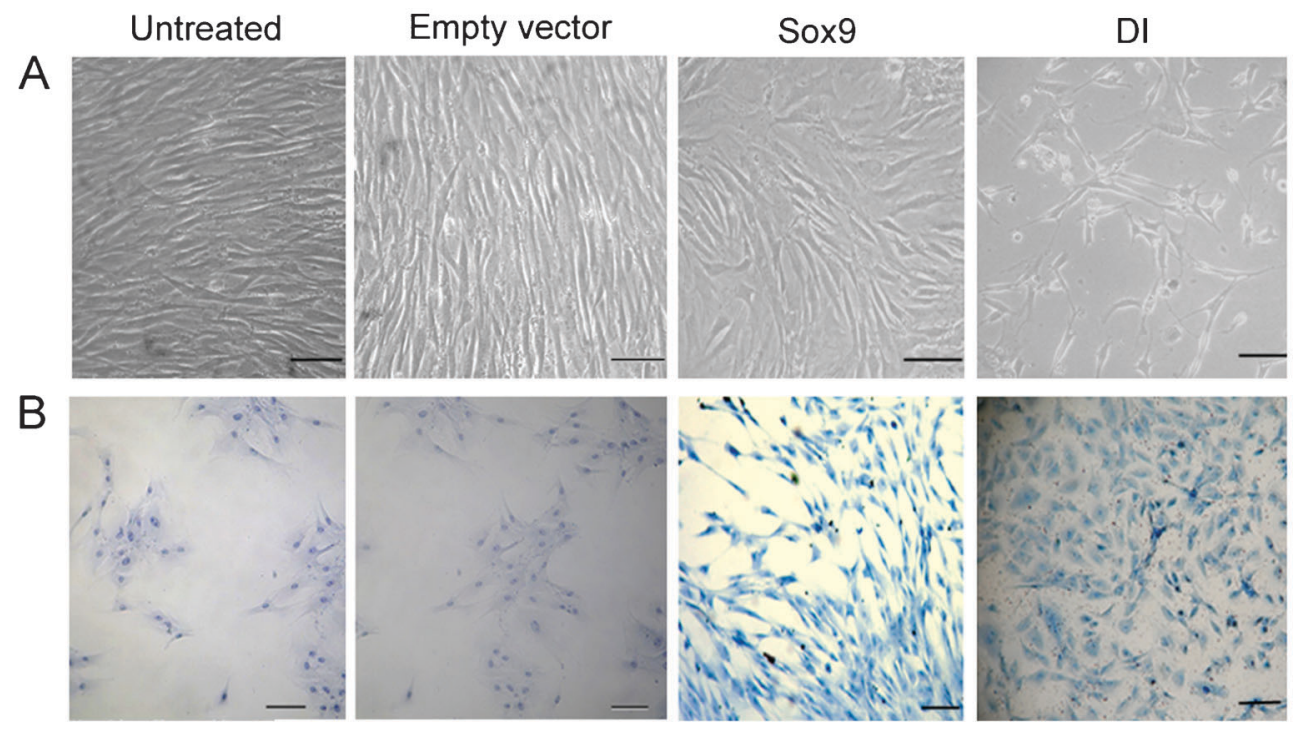

Figure 2. Effects of Sox9 overexpression on morphological changes and proteoglycan disposition in hUC-MSCs. A, Untreated hUCMSCs and those transfected with empty vector or Sox9-expressing plasmid had a similar fibroblast-like morphology. In contrast, hUCMSC cells cultured in the chondrogenic medium (differentiation-induced group: DI) for $48 \mathrm{~h}$ were polygonal or irregular in shape and prone to detach from the plate. Bar $=50 \mu \mathrm{m}$. B. Assessment of proteoglycan disposition by toluidine blue staining. The degree of toluidine blue staining was low in untreated and empty vector-transfected control hUC-MSC cells with basic maintenance medium. After 2-3 weeks of monolayer culture, Sox9-transfected hUC-MSCs and those treated with chondrogenic medium (DI) showed strong toluidine blue staining. Bar $=100 \mu \mathrm{m}$.

\section{Induction of aggrecan and type II collagen expression by Sox 9 overexpression}

RT-PCR analysis revealed elevated mRNA expression of both aggrecan and type II collagen in Sox9-transfected cells vs empty vector-transfected counterparts (Figure 3). Moreover, such elevation was found to be time-dependent for up to 10 days of culture. However, no detectable level of the type I collagen transcript was observed in the Sox9 group. After 2 weeks of culture, cells grown in chondrogenic medium had a significant increase in the mRNA expression of aggrecan and type I and type II collagens compared to untreated control cells.

Immunocytochemistry further demonstrated that genetic delivery of Sox 9 markedly enhanced the expression of aggrecan and type II collagen in hUC-MSCs, compared with empty vector-transfected counterparts (Figure 4). Such enhancement was similar to that seen in the group treated with chondrogenic medium (Figure 4).

\section{Discussion}

Because of easy availability, multilineage differentiation potential, few ethical concerns, and low immunogenicity, MSCs are promising candidates for tissue engineering (21). Although bone marrow is the main source, MSCs have already been isolated from various other tissues, such as adipose tissue (22) and umbilical cord (23). Choudhery et al. (24) reported that confluent cultures of MSCs either from adipose tissue or cord tissue show a fibroblastic morphology. They further demonstrated that the isolated MSCs are positive for CD44, CD73, CD90, and CD105 and negative for the hematopoietic markers CD3, CD14, CD19, CD34, and CD45. Functional studies revealed that MSCs derived from adipose and cord tissue can efficiently differentiate into adipose, bone, cartilage, and neuronal structures (24). In accordance with these findings, we observed that umbilical cord blood-derived cells in monolayer culture had a fibroblast-like morphology. Moreover, these cells displayed phenotypic characteristics typical of MSCs, as evidenced by expression of CD44, CD105, CD73, and CD90 and lack of expression of CD34, CD45, CD19, CD14, and MHC II. Despite similar morphology and molecular phenotype, MSCs from different sources vary in proliferation potential. It has been suggested that umbilical cord blood-derived MSCs have the highest proliferation capacity, followed by adipose tissue-derived MSCs and bone marrow-derived MSCs (24-26). Therefore, umbilical cord blood is an attractive alternative to bone marrow for large-scale production of MSCs.

Genetic modification is a powerful tool to induce committed differentiation of MSCs. We found that transfection with an empty vector or Sox9-expressing plasmid has little influence on the cell morphology of hUCMSCs. In contrast, hUC-MSCs cultured in the chondrogenic medium underwent a morphological change to polygonal or irregular cells and were prone to detach from 


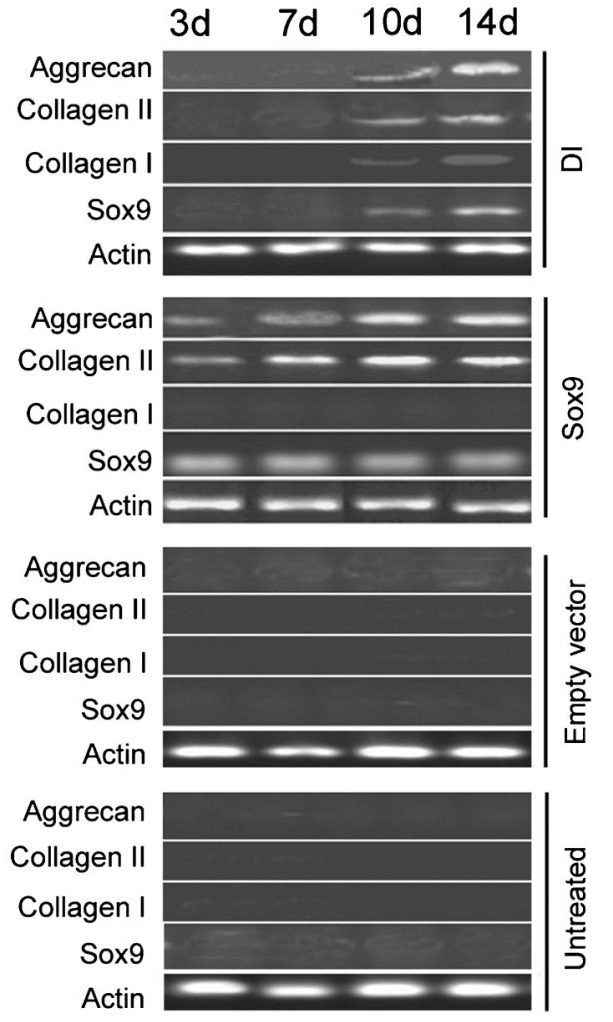

Figure 3. RT-PCR analysis of the mRNA expression of Sox9, collagen I, collagen II, and aggrecan in untreated hUC-MSCs cells and those transfected with empty vector or Sox9-expressing plasmid or induced with the chondrogenic medium (differentiation-induced group: DI). Representative gel photographs of RTPCR products from three independent experiments are shown. d: days. the plate. These findings reflect that gene transfection causes lower toxicity to hUC-MSCs than the addition of chondrogenic growth factors. Numerous molecular factors have been identified as responsible for promoting chondrogenic differentiation of MSCs $(27,28)$. It has been documented that exogenous administration of transforming growth factor-beta 1 (TGF- $\beta 1$ ) efficiently stimulates chondrogenesis of human MSCs in pellet cultures (29). Bone morphogenetic protein (BMP)-4 and BMP-2 are also effective in provoking chondrogenesis of primary human MSCs in pellet culture (28). However, chondrogenesis triggered by BMP-2 and BMP-4 gene transfer showed considerable evidence of hypertrophic differentiation. Sox 9 is a well-established inducer of chondrogenesis, controlling the expression of numerous cartilage ECM components (15). Furumatsu et al. (30) reported that Smad3 overexpression strongly induces the primary chondrogenesis of human MSCs through activation of Sox9-dependent transcription. Silencing of Sox9 using RNA interference technology abrogates TGF- $\beta 1$-induced chondrogenic differentiation of human bone marrow-derived MSCs (31). Our present data confirm the master role of Sox9 in chondrogenesis, as evidenced by the finding that Sox 9 overexpression significantly raised proteoglycan deposition and enhanced the expression of aggrecan and type II collagen in hUC-MSCs. Additionally, we found that Sox9 overexpression significantly inhibited the expression of type I collagen. It has been shown that hypertrophic chondrocytes extensively synthesize type I collagen and type $X$ collagen (32). These findings suggest that short-term genetic delivery of Sox 9 can efficiently direct chondrogenic differentiation of hUC-MSCs, without inducing hypertrophy. However, it has been found that continued expression of Sox9 in differentiated chondrocytes results in subsequent
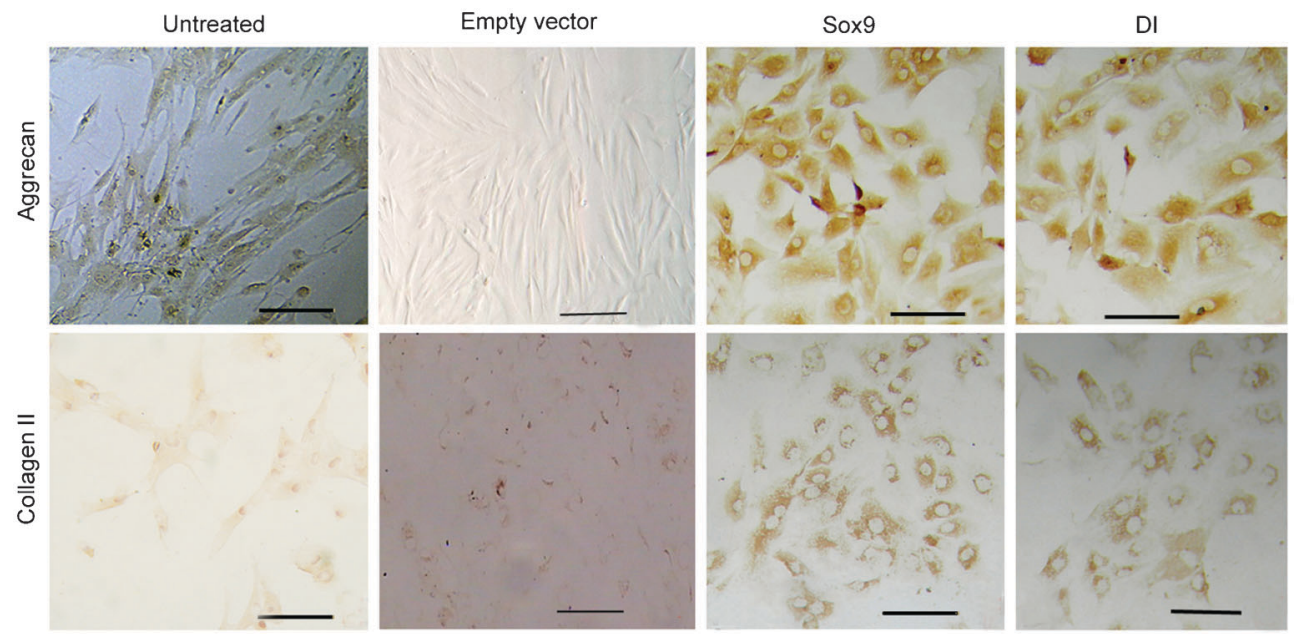

Figure 4. Sox9 overexpression induces chondrogenic differentiation in hUC-MSC cells. For induction of chondrogenic differentiation, cells were treated as described in Material and Methods. After the treatments, cells were subjected to immunocytochemistry for aggrecan and collagen II. Similar to the treatment with chondrogenic medium, genetic delivery of Sox9 markedly enhanced the expression of aggrecan and type II collagen in hUC-MSCs. DI: differentiation-induced group. Bar $=50 \mu \mathrm{m}$. 
hypertrophy (33). Therefore, an inducible gene delivery system may be required for efficient control of Sox 9 expression during the chondrogenesis of MSCs.

Some limitations of this study should be noted. First, there is no information on the impact of Sox9 overexpression on the proliferation and multilineage differentiation potential of MSCs. Second, it remains unclear what may be the long-term effects of sustained expression of Sox9 on the molecular and functional characteristics of MSCs. Finally, there is no mechanistic investigation of Sox9-mediated chondrogenesis of hUC-MSCs.

In conclusion, our results demonstrate that human umbilical cord blood is an important source of MSCs, and enforced expression of Sox 9 accelerates the chondrogenic

\section{References}

1. Redman SN, Oldfield SF, Archer CW. Current strategies for articular cartilage repair. Eur Cell Mater 2005; 9: 23-32.

2. Popa E, Reis R, Gomes M. Chondrogenic phenotype of different cells encapsulated in kappa-carrageenan hydrogels for cartilage regeneration strategies. Biotechnol Appl Biochem 2012; 59: 132-141, doi: 10.1002/bab.1007.

3. Oldershaw RA. Cell sources for the regeneration of articular cartilage: the past, the horizon and the future. Int J Exp Pathol 2012; 93: 389-400.

4. Schulze-Tanzil G. Activation and dedifferentiation of chondrocytes: implications in cartilage injury and repair. Ann Anat 2009; 191: 325-338, doi: 10.1016/j.aanat.2009.05.003.

5. Kock L, van Donkelaar CC, Ito K. Tissue engineering of functional articular cartilage: the current status. Cell Tissue Res 2012; 347: 613-627, doi: 10.1007/s00441-011-1243-1.

6. Pittenger MF, Mackay AM, Beck SC, Jaiswal RK, Douglas $\mathrm{R}$, Mosca JD, et al. Multilineage potential of adult human mesenchymal stem cells. Science 1999; 284: 143-147, doi: 10.1126/science.284.5411.143.

7. Qian Q, Qian H, Zhang X, Zhu W, Yan Y, Ye S, et al. 5Azacytidine induces cardiac differentiation of human umbilical cord-derived mesenchymal stem cells by activating extracellular regulated kinase. Stem Cells Dev 2012; 21: 6775, doi: 10.1089/scd.2010.0519.

8. Yuan J, Huang G, Xiao Z, Lin L, Han T. Overexpression of beta-NGF promotes differentiation of bone marrow mesenchymal stem cells into neurons through regulation of AKT and MAPK pathway. Mol Cell Biochem 2013; 383: 201-211, doi: 10.1007/s11010-013-1768-6.

9. Williams AR, Hare JM. Mesenchymal stem cells: biology, pathophysiology, translational findings, and therapeutic implications for cardiac disease. Circ Res 2011; 109: 923940, doi: 10.1161/CIRCRESAHA.111.243147.

10. Liang L, Dong C, Chen X, Fang Z, Xu J, Liu M, et al. Human umbilical cord mesenchymal stem cells ameliorate mice trinitrobenzene sulfonic acid (TNBS)-induced colitis. Cell Transplant 2011; 20: 1395-1408, doi: 10.3727/096368910X557245.

11. Cho PS, Messina DJ, Hirsh EL, Chi N, Goldman SN, Lo DP, et al. Immunogenicity of umbilical cord tissue derived cells. Blood 2008; 111: 430-438, doi: 10.1182/blood-2007-03078774. differentiation of hUC-MSCs. Therefore, Sox9-based genetic modification of hUC-MSCs may be an attractive cell source for cartilage tissue engineering.

\section{Supplementary material}

Click here to view [pdf].

\section{Acknowledgments}

Research supported by the Natural Science Foundation of China (\#81000416), the Fundamental Research Funds for the Central Universities (2011), and Funds from the Second Hospital of Xi'an Jiaotong University (\#RC201102, \#YJ(ZD)201301) of China.

12. Elsler S, Schetting S, Schmitt G, Kohn D, Madry H, Cucchiarini M. Effective, safe nonviral gene transfer to preserve the chondrogenic differentiation potential of human mesenchymal stem cells. J Gene Med 2012; 14: 501-511, doi: 10.1002/jgm.2644.

13. Bi W, Deng JM, Zhang Z, Behringer RR, de Crombrugghe B. Sox9 is required for cartilage formation. Nat Genet 1999; 22: $85-89$, doi: $10.1038 / 8792$.

14. Cucchiarini M, Thurn T, Weimer A, Kohn D, Terwilliger EF, Madry $H$. Restoration of the extracellular matrix in human osteoarthritic articular cartilage by overexpression of the transcription factor SOX9. Arthritis Rheum 2007; 56: 158167, doi: 10.1002/art.22299.

15. Kawakami Y, Tsuda M, Takahashi S, Taniguchi N, Esteban $\mathrm{CR}$, Zemmyo M, et al. Transcriptional coactivator PGC-1 alpha regulates chondrogenesis via association with Sox9. Proc Natl Acad Sci U S A 2005; 102: 2414-2419, doi: 10.1073/ pnas.0407510102.

16. Venkatesan JK, Ekici M, Madry H, Schmitt G, Kohn D, Cucchiarini M. SOX9 gene transfer via safe, stable, replication-defective recombinant adeno-associated virus vectors as a novel, powerful tool to enhance the chondrogenic potential of human mesenchymal stem cells. Stem Cell Res Ther 2012; 3: 22, doi: 10.1186/scrt113.

17. Jin HJ, Bae YK, Kim M, Kwon SJ, Jeon HB, Choi SJ, et al. Comparative analysis of human mesenchymal stem cells from bone marrow, adipose tissue, and umbilical cord blood as sources of cell therapy. Int J Mol Sci 2013; 14: 1798618001, doi: 10.3390/ijms140917986.

18. Yao TH, Yang ZQ, Wang ZH, Tu JB, Ma JM. Inducing differentiation of human umbilical cord blood mesenchymal stem cells into chondroblast in vitro. Chin J Aesth Med 2007; 16: $450-454$.

19. Wang ZH, Yang ZQ, He XJ, Kamal BE, Xing Z. Lentivirusmediated knockdown of aggrecanase-1 and -2 promotes chondrocyte-engineered cartilage formation in vitro. Biotechnol Bioeng 2010; 107: 730-736, doi: 10.1002/bit. 22862.

20. Wang ZH, Yang ZQ, He XJ, Wang L, Li LX, Tu JB. Effects of RNAi-mediated inhibition of aggrecanase-1 and aggrecanase2 on rat costochondral chondrocytes in vitro. Acta Pharmacol 
Sin 2008; 29: 1215-1226, doi: 10.1111/j.1745-7254.2008. 00856.x

21. Jung $S$, Panchalingam KM, Wuerth RD, Rosenberg L, Behie LA. Large-scale production of human mesenchymal stem cells for clinical applications. Biotechnol Appl Biochem 2012; 59: 106-120, doi: 10.1002/bab.1006.

22. Wlodarski KH, Wlodarski $\mathrm{P}$, Galus R, Mazur S. Adipose mesenchymal stem cells. Their characteristics and potential application in tissue repair. Pol Orthop Traumatol 2012; 77: 97-99.

23. Patel AN, Vargas V, Revello P, Bull DA. Mesenchymal stem cell population isolated from the subepithelial layer of umbilical cord tissue. Cell Transplant 2013; 22: 513-519, doi: $10.3727 / 096368912 \times 655064$.

24. Choudhery MS, Badowski M, Muise A, Harris DT. Comparison of human mesenchymal stem cells derived from adipose and cord tissue. Cytotherapy 2013; 15: 330343, doi: 10.1016/j.jcyt.2012.11.010.

25. Peng L, Jia Z, Yin X, Zhang $X$, Liu $Y$, Chen $P$, et al. Comparative analysis of mesenchymal stem cells from bone marrow, cartilage, and adipose tissue. Stem Cells Dev 2008; 17: 761-773, doi: 10.1089/scd.2007.0217.

26. Kern S, Eichler H, Stoeve J, Kluter H, Bieback K. Comparative analysis of mesenchymal stem cells from bone marrow, umbilical cord blood, or adipose tissue. Stem Cells 2006; 24: 1294-1301, doi: 10.1634/stemcells.20050342.

27. Steinert AF, Palmer GD, Pilapil C, Noth U, Evans $\mathrm{CH}$, Ghivizzani SC. Enhanced in vitro chondrogenesis of primary mesenchymal stem cells by combined gene transfer. Tissue Eng Part A 2009; 15: 1127-1139, doi: 10.1089/ten.tea. 2007.0252.
28. Steinert AF, Proffen B, Kunz M, Hendrich C, Ghivizzani SC, Noth $\mathrm{U}$, et al. Hypertrophy is induced during the in vitro chondrogenic differentiation of human mesenchymal stem cells by bone morphogenetic protein- 2 and bone morphogenetic protein-4 gene transfer. Arthritis Res Ther 2009; 11: R148, doi: 10.1186/ar2822.

29. Kawamura K, Chu CR, Sobajima S, Robbins PD, Fu FH, Izzo NJ, et al. Adenoviral-mediated transfer of TGF-beta1 but not IGF-1 induces chondrogenic differentiation of human mesenchymal stem cells in pellet cultures. Exp Hematol 2005; 33: 865-872, doi: 10.1016/j.exphem.2005.05.010.

30. Furumatsu T, Tsuda M, Taniguchi N, Tajima Y, Asahara H. Smad3 induces chondrogenesis through the activation of SOX9 via CREB-binding protein/p300 recruitment. J Biol Chem 2005; 280: 8343-8350, doi: 10.1074/jbc.M41391 3200.

31. Chang $Y$, Ueng SW, Lin-Chao S, Chao CC. Involvement of Gas7 along the ERK1/2 MAP kinase and SOX9 pathway in chondrogenesis of human marrow-derived mesenchymal stem cells. Osteoarthritis Cartilage 2008; 16: 1403-1412, doi: 10.1016/j.joca.2008.03.018.

32. Galotto M, Campanile G, Robino G, Cancedda FD, Bianco $P$, Cancedda R. Hypertrophic chondrocytes undergo further differentiation to osteoblast-like cells and participate in the initial bone formation in developing chick embryo. J Bone Miner Res 1994; 9: 1239-1249, doi: 10.1002/jbmr. 5650090814

33. Ikegami D, Akiyama $H$, Suzuki A, Nakamura $T$, Nakano $T$, Yoshikawa $H$, et al. Sox9 sustains chondrocyte survival and hypertrophy in part through Pik3ca-Akt pathways. Development 2011; 138: 1507-1519, doi: 10.1242/ dev.057802. 